Original Article

\title{
SIMPLE METHOD FOR OPTIMIZATION OF HEPARIN AND ENOXAPARIN DETECTION USING AGAROSE GEL ELECTROPHORESIS
}

\author{
AHMAD A. ALMEMAN \\ Pharmacology Department, College of Medicine, Qassim, Qassim University, PoBox 54, Code 51911 \\ Email: ahmadalmeman@gmail.com
}

Received: 26 Dec 2018 Revised and Accepted: 15 Feb 2019

\begin{abstract}
Objective: A simple gel electrophoresis method for low-molecular-weight heparins (LMWH) is required for use in a variety of laboratories to allow further identification and purification. This study aimed to optimize the detection of heparin and enoxaparin (low-molecular-weight heparin by gel electrophoresis.

Methods: Several gel electrophoresis conditions were tested to optimize the detection of enoxaparin by using a simple method with a modified Volpi's approach. Multiple gel thicknesses, voltage settings, and enoxaparin concentrations were tested in the optimization procedure. Enoxaparin was purchased from a local supplier as pre-filled pharmaceutical injections. Highly purified $0.5 \%$ and $1.0 \%$ agarose gels were prepared and a series of enoxaparin concentrations was added to both gels for comparison and optimization. The $0.2 \%$ toluidine blue stain was prepared by the addition of $1 \mathrm{ml}$ in an ethanol-water-acetic acid mixture (50:49:1; v/v/w). The staining process comprised two steps: first, toluidine blue was added for 30 min and destained overnight in the solvent mixture. Subsequently, the following morning, the second step was conducted, in which the gel was restained for $30 \mathrm{~min}$ with the same concentration of toluidine blue. We continued to stain the gel until the bands were visible.
\end{abstract}

Results: The gel electrophoresis results showed that clearest and sharpest bands were obtained using 65-75 mAh and $85 \mathrm{~V}$ settings. At $95 \mathrm{mAh}$, the bands were slightly washed out.

Conclusion: This study successfully facilitated the detection of enoxaparin, a LMWH, and heparin in the laboratory by using simple tools and techniques available in most laboratories.

Keywords: Gel electrophoresis, Low-molecular-weight heparin, Method optimization, toluidine blue

(C) 2019 The Authors. Published by Innovare Academic Sciences Pvt Ltd. This is an open access article under the CC BY license (http://creativecommons.org/licenses/by/4.0/) DOI: http://dx.doi.org/10.22159/ijpps.2019v11i4.31655

\section{INTRODUCTION}

Crude heparin contains several impurities and requires purification before it is ready for the pharmaceutical market [1]. The most common impurities are dermatan sulfate (DS) and chondroitin sulfate (CS). Several electrophoretic methods have been used to detect the purity and quality of heparin, such as capillary electrophoresis (CE), polyacrylamide gel electrophoresis (PAGE), and agarose gel electrophoresis [2]. In addition to electrophoretic assays, sophisticated methods are also essential to receive approval US Food and Drug Administration (FDA), such as nuclear magnetic resonance (NMR) and liquid chromatography-mass spectrometry (LC-MS) [3]. Heparin is a heterogeneous molecule that exists as molecules of variable molecular mass, ranging from 3,000 to 40,000 Da. However, low-molecularweight heparins (LMWH) contain less variation in mass and are more purified in the manufacturing process than other heparins [4]. As LMWH is a degradation product of heparin and is more purified, it is easier to detect on a gel than unfractionated heparin (UFH) $[2,4]$.

CE is a validated and sensitive technique for the separation of sulfated and non-sulfated glycosaminoglycans (GAGs). The limitations of this method include wide peaks, partial separation of contaminants, and limited resolution [5]. Several studies have applied further modifications of this method to enhance its reliability, sensitivity, and resolution for impurity separation [6-8]. PAGE is another essential method for the characterization of UFH and LMWH and is valuable for purifying the over-sulfated CS (OSCS) impurities [9]. Similarly, the PAGE method has been subjected to several improvements and developments to enhance it's precision and reliability [10]. In contrast, although agarose gel electrophoresis is a simple and widely used approach, only a few researchers have utilized the technique for heparin purification [11]. In general, agarose gel electrophoresis can be used to separate the main impurities, such as OSCS, DS, and CS, by using high-voltage electrophoresis overnight. Furthermore, gel electrophoresis can separate UFH and LMWH in simple steps at a reasonably low cost [12]. The advantage of this method is its availability and accessibility by most laboratories. This study aimed to optimize the detection of enoxaparin, low-molecular-weight heparin, by using simple electrophoretic steps with a simple and cheap staining process.

\section{MATERIALS AND METHODS}

Several gel electrophoresis procedures were performed to test and optimize the detection of enoxaparin using a simple modification in the approach by Volpi [12]. This method was developed to simplify the detection of LMWH by using a gel. Therefore, several gel thicknesses, voltage settings, and enoxaparin concentrations were tested in the optimization procedure. Enoxaparin was purchased from a local supplier as pre-filled pharmaceutical injections. The electrophoresis cell instruments were prepared. Highly purified $0.5 \%$ and $1 \%$ agarose gels were prepared for the comparison. Both gels were immersed in $0.04 \mathrm{M}$ barium acetate buffer at a $\mathrm{pH}$ of approximately 5.8. Serial dilutions of enoxaparin were added to both gels for comparison and optimization. All enoxaparin samples were layered into the cells at a volume of $0.5 \mu \mathrm{l} /$ cell. A buffer of $0.05 \mathrm{M}$ 1,2-diaminopropane was added, followed by acetic acid to adjust the $\mathrm{pH}$ to 9.0. The current was applied for $15 \mathrm{~min}$ at $4{ }^{\circ} \mathrm{C}$, at $65,75,85$, and $95 \mathrm{mAh}$ and $85 \mathrm{~V}$. After electrophoresis, the gels were immersed in $0.2 \%$ cetylpyridinium chloride for $3 \mathrm{~h}$ as part of the washing process. The toluidine blue stain was prepared at $0.2 \%$ in $1 \mathrm{ml}$ of a mixture of ethanol-water-acetic acid (50:49:1; v/v/w). The staining process was completed in two steps: first, toluidine blue was added for $30 \mathrm{~min}$, followed by destaining with a $0.2 \%$ ethanol-water-acetic acid (50:49:1; v/v/w) solution overnight. The following morning, the gel was restained for another $30 \mathrm{~min}$ using the same concentration of toluidine blue. The staining process was continued until clear bands were visible on the gel. The analysis was performed by using a densitometer scanner (Macintosh IIsi with Microtek International Interface).

\section{RESULTS}

Optimal conditions were identified from the electrophoresis of the solutions in different thicknesses of gels, currents, voltages, and heparin concentrations. 
Table 1: Electrophoresis materials

\begin{tabular}{ll}
\hline Item & Manufacturer \\
\hline Agarose gel & Sigma-Aldrich, USA \\
Barium acetate & Panreac Chemicals, Barcelona, Spain \\
Electrophoresis apparatus & C. B. S. Scientific Co., CA, USA \\
Hexadecyltrimethylammonium bromide & Sigma-Aldrich, UK \\
Toluidine blue & LOBA Chemie, India \\
Ethanol & Fisher Scientific, UK \\
Acetic acid & Winlab, UK \\
Clexane & Sanofi Aventis, France \\
\hline
\end{tabular}

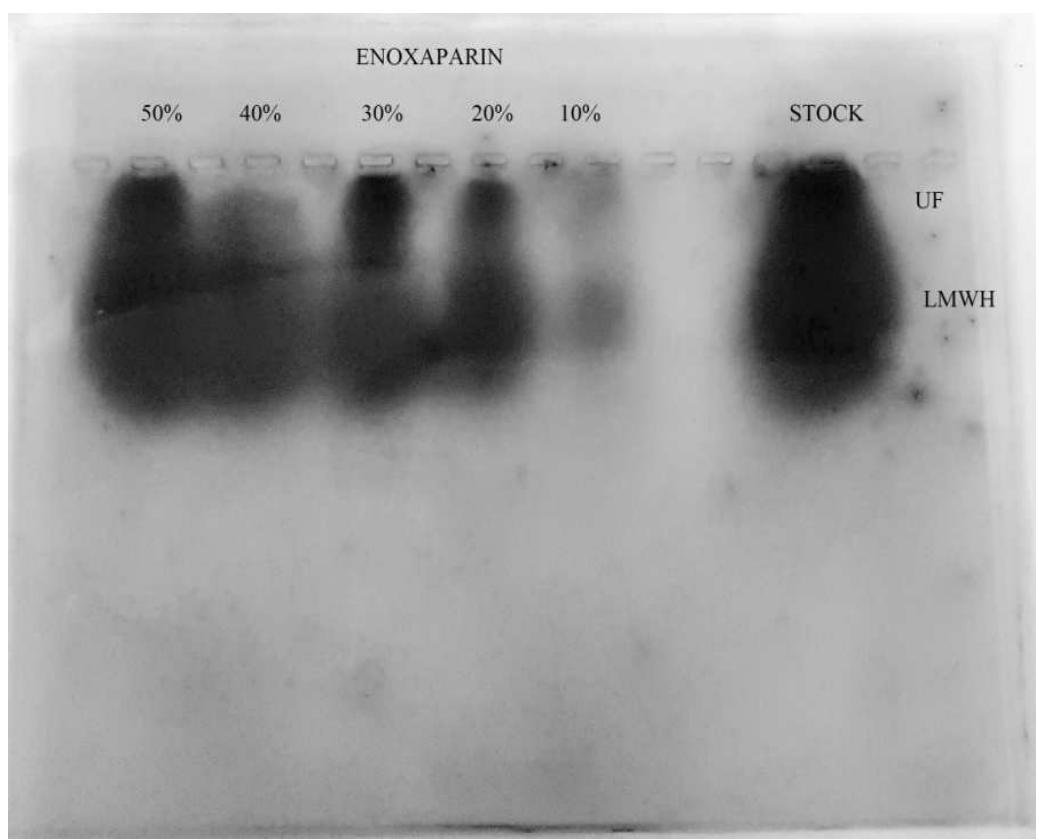

Fig. 1: Comparison of different enoxaparin and heparin concentrations resolved by $0.5 \%$ agarose gel electrophoresis at $85 \mathrm{~V}$ and $65 \mathrm{~mA}$. The stock solution at $\mathbf{1 0 0 \%}$ concentration was added as a standard. UFH, unfractionated heparin; LMWH, low-molecular-weight heparin

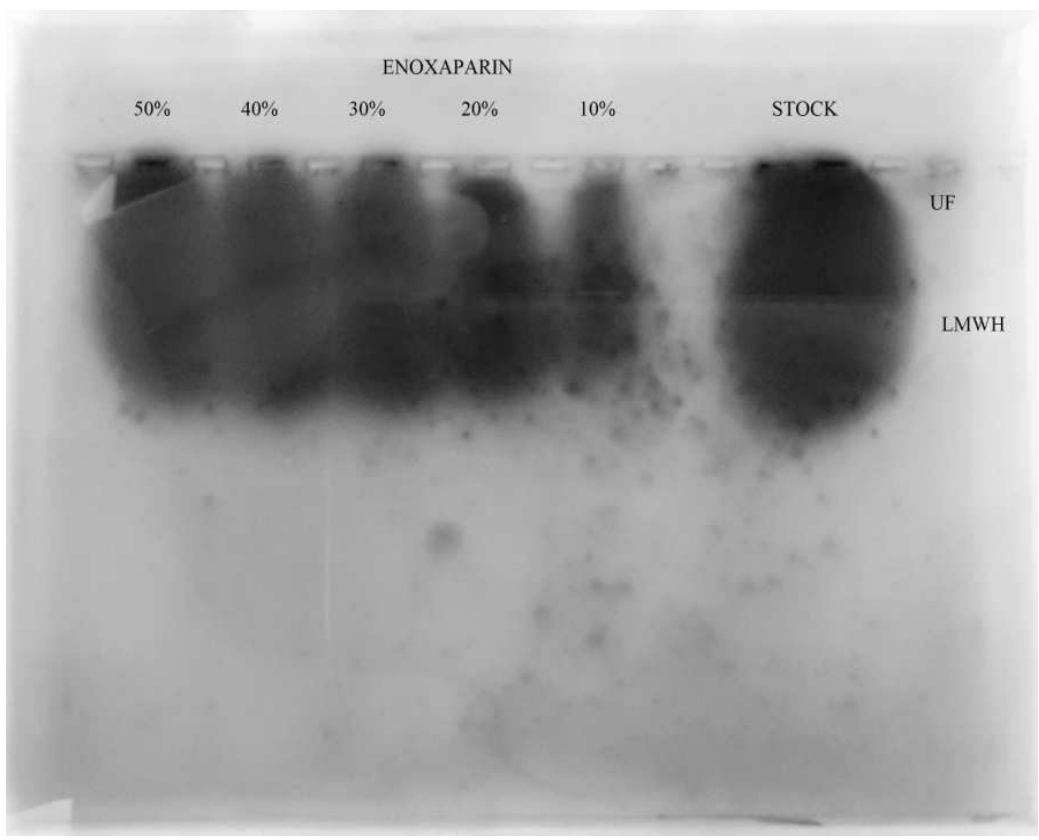

Fig. 2: Comparison of different enoxaparin and heparin concentrations resolved by $1 \%$ agarose gel electrophoresis at $85 \mathrm{~V}$ and $65 \mathrm{~mA}$ The stock solution of $100 \%$ concentration was added as the standard solution. UFH, unfractionated heparin; LMWH, low-molecularweight heparin 


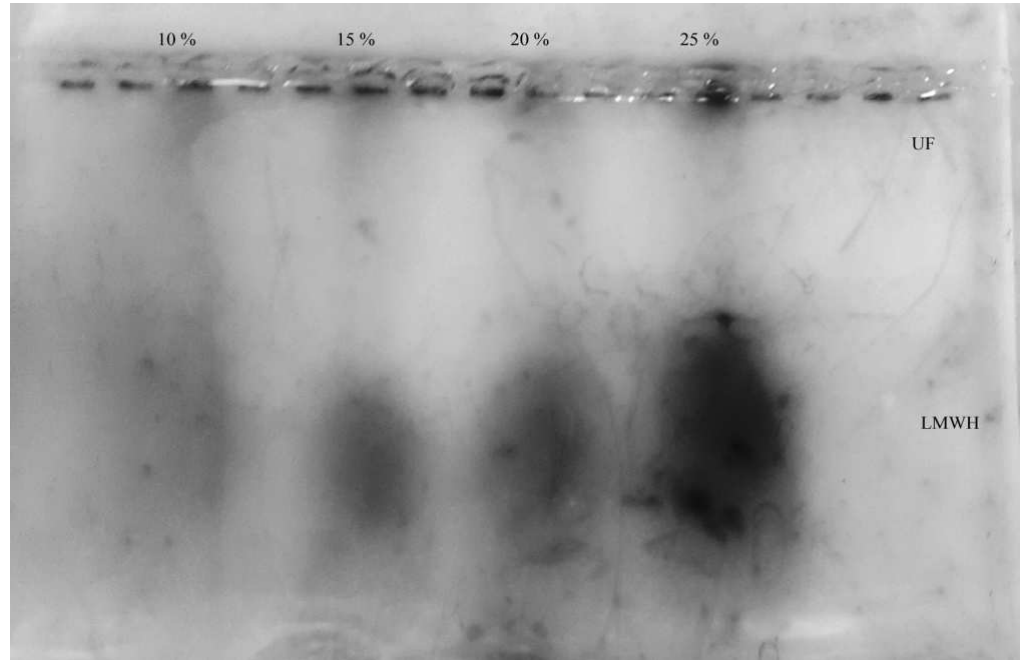

Fig. 3: Comparison of different enoxaparin concentrations resolved by $1 \%$ agarose gel electrophoresis at $85 \mathrm{~V}$ and $75 \mathrm{~mA}$. UFH, unfractionated heparin; LMWH, low-molecular-weight heparin

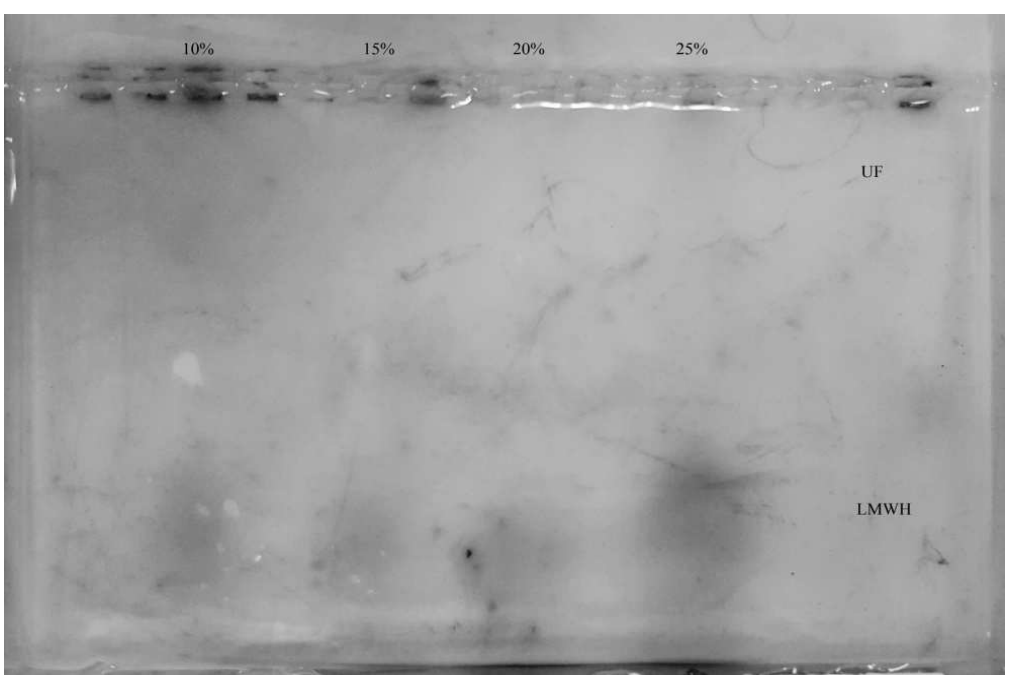

Fig. 4: Comparison of different enoxaparin concentrations resolved by $1 \%$ agarose gel electrophoresis at $85 \mathrm{~V}$ and $85 \mathrm{~mA}$. UFH, unfractionated heparin; LMWH, low-molecular-weight heparin

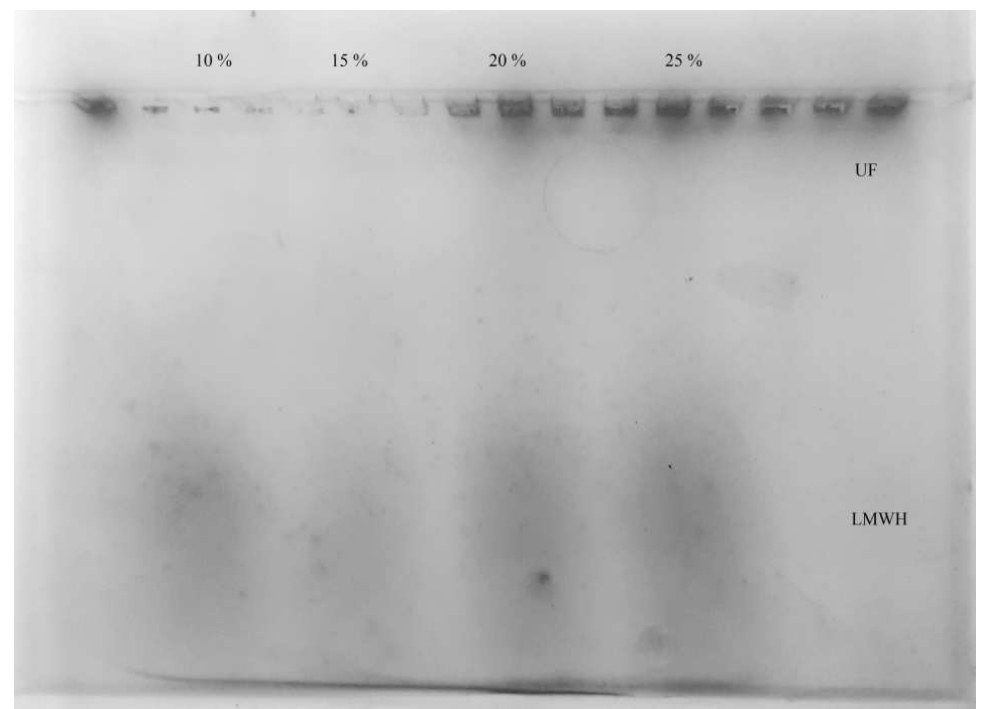

Fig. 5: Comparison of different enoxaparin concentrations resolved by $1 \%$ agarose gel electrophoresis at $85 \mathrm{~V}$ and $95 \mathrm{~mA}$. UFH, unfractionated heparin; LMWH, low-molecular-weight heparin 


\section{DISCUSSION}

A simple gel electrophoresis method to allow further identification and purification of LMWH is required in numerous laboratories. In this study, we successfully established a simplified detection method for enoxaparin (a low-molecular-weight heparin) and heparin in the laboratory by using simple tools and techniques that are widely available in most laboratories. Although other gel-based methods have been approved for heparin identification, such as capillary electrophoresis, a simple gel agarose electrophoresis method would be an indispensable tool [3].

Gel thickness plays an important role in the performance of gel electrophoresis for the detection of various substances. Most published data used $0.5 \%$ highly purified gels [13] for the detection of heparin and LMWH; as we did not find any differences between $0.5 \%$ and $1 \%$ gels, the use of $0.5 \%$ gels would be more cost-effective. Ideally, higher enoxaparin concentrations create wider, overlapping bands (fig. 1 and 2). Extremely low concentrations, such as those $\leq 10 \%$, may generate very bright bands if the procedure is not performed correctly. Thus, a concentration range of $20 \%-25 \%$ is a more reasonable choice and provides clear non-overlapping bands. Other studies have used different concentrations based on the provided standards $[1,12]$.

The applied voltage and time are both essential to a successful separation and in this study, the separation of enoxaparin was mainly evaluated at $85 \mathrm{~V}$ and $65-100 \mathrm{mAh}$. We also investigated the separation at 75,85 , and $95 \mathrm{mAh}$. At 65-85 $\mathrm{mAh}$, all the bands appeared to be well separated and the most efficient separation was observed at $85 \mathrm{mAh}$. This allowed the bands to shift toward the end of the gel, which might not be necessary. At $95 \mathrm{mAh}$, some bands were washed out of the gel; thus, this voltage was unsuitable. Other studies have reported that full separation was achieved at $100 \mathrm{mAh}$, but the exact running voltage was not specified.

Staining is the final step, but several studies have failed to detect LMWH and heparin using agarose gel electrophoresis with the most commonly used dye, toluidine blue. However, others succeeded easily [11]. Thus, the sequential use of other dyes has been proposed following toluidine blue, such as Stains-all, especially when GAGs are unavailable [12]. In this study, we did not detect any bands after the first staining process and therefore we repeated the same staining process with the expectation that the bands would be clear. This is the first study to report the successful use of repeated staining with toluidine blue with various gel processing conditions.

\section{CONCLUSION}

Agarose gel electrophoresis is a simple and widely available method that can be used for the detection and purification of enoxaparin and heparin. Repeated (double) staining with toluidine blue may enhance the band clarity.

\section{ACKNOWLEDGMENT}

We would like to thank Qassim University for providing the chemicals and laboratories. Special thanks go to Mr. Casimero A. Victoria Jr., a key member of these laboratories.

\section{CONFLICT OF INTERESTS}

We have no conflicts of interest to declare

\section{REFERENCES}

1. Liu H, Zhang Z, Linhardt RJ. Lessons learned from the contamination of heparin. Nat Prod Rep 2009;26:313-21.

2. Volpi N, Maccari F, Suwan J, Linhardt RJ. Electrophoresis for the analysis of heparin purity and quality. Electrophoresis 2012;33:1531-7.

3. Guerrini M, Beccati D, Shriver Z, Naggi A, Viswanathan K, Bisio A, et al. Oversulfated chondroitin sulfate is a contaminant in heparin associated with adverse clinical events. Nat Biotechnol 2008;26:669-75.

4. Michael S, Conners III MD, Samuel R, Money MD. The new heparins. Ochsner J 2002;4:41-7.

5. Volpi N, Maccari F. Electrophoretic approaches to the analysis of complex polysaccharides. J Chromatogr B 2006;834:1-13.

6. Somsen GW, Tak YH, Torano JS, Jongen PM, de Jong GJ. Determination of oversulfated chondroitin sulfate and dermatan sulfate impurities in heparin by capillary electrophoresis. J Chromatogr A 2009;1216:4107-12.

7. Tripodi V, Flor S, Dobrecky C, Contin M, Lucangioli S. Novel and highly sensitive mixed-polymeric electrokinetic chromatography system for determination of contaminants and impurities of heparin samples. Electrophoresis 2010;31:3606-12.

8. Volpi N, Maccari F, Linhardt RJ. Quantitative capillary electrophoresis determination of oversulfated chondroitin sulfate as a contaminant in heparin preparations. Anal Biochem 2009;388:140-5.

9. Zhang Z, Li B, Suwan J, Zhang F, Wang Z, Liu H, et al. Analysis of pharmaceutical heparins and potential contaminants using $1 \mathrm{H}-$ NMR and PAGE. J Pharm Sci 2009;98:4017-26.

10. Zhang Z, Weiwer M, Li B, Kemp MM, Daman TH, Linhardt RJ. Oversulfated chondroitin sulfate: impact of a heparin impurity, associated with adverse clinical events, on low-molecularweight heparin preparation. J Med Chem 2008;51:5498-501.

11. Volpi N, Buzzega D. Agarose-gel electrophoresis for the quality assurance and purity of heparin formulations. J Pharm Biomed Anal 2012;67-68:144-7.

12. Volpi N, Maccari F. Detection of submicrogram quantities of glycosaminoglycans on agarose-gels by sequential staining with toluidine blue and Stains-all. Electrophoresis 2002;23:4060-6.

13. Sarwar MI, Hussain MS, Manzoor MA, Ahmad M, Hakeem A. Isolation and purification of heparin from bovine pancreas by different methods. JSZMC 2015;6:873-7.

14. Warda M, Gouda EM, Toida T, Chic L, Linhardt RJ. Isolation and characterization of raw heparin from dromedary intestine: evaluation of a new source of pharmaceutical heparin. Comp Biochem Physiol C Toxicol Pharmacol 2003;136:357-65. 